\title{
Somatic mutation profiling of hobnail variant of papillary thyroid carcinoma
}

\author{
Luca Morandi', Alberto Righi2, Francesca Maletta3, Paola Rucci4, Fabio Pagni5, \\ Marco Gallo6, Sabrina Rossi7, Leonardo Caporali, Anna Sapino9, \\ Ricardo V Lloyd 10 and Sofia Asioli1
}

'Department of Biomedical and Neuromotor Sciences, Section of Anatomic Pathology 'M. Malpighi' at Bellaria Hospital, University of Bologna, Bologna, Italy 2Department of Pathology, Rizzoli Institute, (IRCCS), Bologna, Italy ${ }^{3}$ Department of Medical Sciences, University of Turin, Turin, Italy 4Section of Hygiene and Biostatistics, University of Bologna, Bologna, Italy ${ }^{5}$ Department of Pathology, University of Milano Bicocca, Monza, Italy ${ }^{6}$ Oncological Endocrinology Unit, Department of Medical Sciences, AOU Città della Salute e della Scienza di Torino, University of Turin, Turin, Italy ${ }^{7}$ Department of Pathology, Regional Hospital, Treviso, Italy 8Istituto delle Scienze Neurologiche di Bologna (IRCCS), Bellaria Hospital, Bologna, Italy Institute for Cancer Research and Treatment (IRCCS), Candiolo, Italy 10University of Wisconsin School of Medicine and Public Health, Madison, Wisconsin, USA

Correspondence should be addressed to S Asioli Email sofia.asioli3@unibo.it

\begin{abstract}
Hobnail variant of papillary thyroid carcinoma (HPTC) represents a recently described, aggressive and rare group of thyroid tumors with poorly understood pathogenesis. Molecular data about this group of cancers are few, and a more detailed molecular characterization of these tumors is needed. The main objective of the study is to define a comprehensive molecular typing of HPTC. Eighteen patients affected by HPTC, including eighteen primary tumors and four lymph node metastases, were screened for NRAS, KRAS, HRAS, BRAF, TP53, PIK3CA, hTERT, PTEN, CDKN2A, EGFR, AKT1, CTNNB1 and NOTCH1 gene mutations. Sequencing is conducted on the MiSEQ system, and molecular data are compared with clinical-pathologic data and follow-up. The patients include 14 women and 4 men. Ages range from 23 to 87 years. All 18 primary tumors of HPTC showed $\geq 30 \%$ hobnail features. BRAF and TP53 mutations are by far the most common genetic alterations in primary HPTC $(72.2 \%$ and $55.6 \%$, respectively), followed by $h T E R T$ (44.4\%), PIK3CA (27.8\%), CTNNB1 (16.7\%), EGFR (11.1\%), AKT1 (5.5\%) and NOTCH1 $(5.5 \%)$. The mutational pattern in primary tumors and metastasis was usually maintained. Univariate Cox regression analyses with bootstrap procedure indicated a significantly increased mortality risk in patients harboring $B R A F$ mutation and $B R A F$ mutation associated with TP53 and/or PIK3CA mutations. The detection of these multiple mutations appears to allow the identification of a subset of more aggressive tumors within the group and to bear information that should be useful for prognostic stratification of these patients including the planning of adjuvant therapy.
\end{abstract}

Endocrine-Related Cancer (2017) 24, 107-117

\section{Introduction}

Thyroid cancer is the most common type of endocrine malignancy. The vast majority of thyroid tumors originate from thyroid follicular cells and encompasses well-differentiated papillary carcinoma and follicular carcinoma. Its prognosis is generally favorable, especially in well-differentiated thyroid carcinomas (DTCs) such

Published by Bioscientifica Ltd 
as papillary and follicular variants, which show survival rates of approximately $95 \%$ at 40 years (LiVolsi et al. 2004). Despite that, $15-20 \%$ of DTCs became radioiodine refractory (RAI-R) and until now no other therapeutic options have been effective (Haugen et al. 2016).

Recently, a new variant of papillary thyroid carcinoma (PTC), named 'hobnail variant' (HPTC) was described (Motosugi et al. 2009, Asioli et al. 2010, 2014, Bellevicine et al. 2012, Kakudo et al. 2012, Lino-Silva et al. 2012, Yang et al. 2013, Schwock et al. 2015, Ieni et al. 2016). It has a prevalence $<2 \%$ and usually behaves more aggressively than classical PTC (Lino-Silva et al. 2012, Asioli et al. 2013, Amacher et al. 2015). The local recurrence rate is $23 \%$, lymph node metastasis rate ranges from $60 \%$ to $75 \%$ and distant metastasis (including lung, brain and bone) rate ranges from $25 \%$ to $40 \%$ (Asioli et al. 2010, 2013, Chung et al. 2013). Overall survival rates are 69.1\% and $64.1 \%$ at 5 and 10 years, respectively (Asioli et al. 2010, 2013, Chung et al. 2013). Disease-specific survival rates are 83,71 and $54 \%$ at 5, 10 and 20 years after surgery, respectively (Lee et al. 2015).

Patients affected by HPTC seem to be good candidates for target therapies because they usually have locally advanced disease and distant metastases at the time of presentation and because the HPTC cells seem to be scarcely responsive to 131I treatment (Haugen et al. 2016).

To date, in PTC, the common known mutations are represented by several RET/PTC rearrangements (Romei \& Elisei 2012), BRAF p.V600 mutation (Mathur et al. 2011, Romei \& Elisei 2012) and more rarely gene amplifications and copy number gains involve genes encoding tyrosine kinase receptors (TKRs) (Abubaker et al. 2008, Liu et al. 2008, Santarpia et al. 2008) and epigenetic pathways including the aberrant methylation of thyroid-specific genes involved in iodine metabolism and/or of tumor suppressor genes, leading to the loss of radioiodine avidity and increased tumor growth, invasion and metastasization (Xing et al. 2015, Viola et al. 2016). Based on increase in our understanding of the molecular mechanisms underlying thyroid carcinogenesis, including that more aggressive thyroid cancers often show more than one gene alteration (Sobrinho-Simoes et al. 2008, Smallridge et al. 2009), innovative target therapies have been proposed in the last decade.

To date, molecular data in HPTCs are few, possibly reflecting the rarity of this entity. However, some previous reports have identified BRAF p.V600E mutation and a few have found RET/PTC1 rearrangements consistent with PTC that has papillary architecture (Bellevicine et al. 2012, Yang et al. 2013, Asioli et al. 2014, Lubitz et al. 2014,
Ieni et al. 2016). A distinct molecular pathway of progression is poorly understood, and there is the need for a more detailed molecular characterization of these tumors. In this study, we report the results of our analysis of molecular alterations in a series of HPTCs that seem to improve risk stratification of these patients and to suggest the possible use of combined conventional and targeted therapies in this new entity.

\section{Materials and methods}

\section{Ethics statement}

All clinical investigations have been conducted according to the principles expressed in the Declaration of Helsinki. The study was approved by local ethics committee (Bologna-Imola CE-BI, number of study: 269/CE 2016 March 29th; Cod. CE: 16014). All information regarding the human material used in this study was managed using anonymous numerical codes.

\section{Sample collection}

Eighteen patients affected by HPTC, including eighteen primary tumors and four lymph node metastases, were selected from the databases of the Divisions of Pathology of the University of Turin (8 patients), University of Wisconsin School of Medicine and Public Health, Madison, Wisconsin (2 patients), University of Milan (3 patients) and University of Bologna (5 patients retrieved from consult cases to SA) and included in the study.

The present series is constituted by previously unreported tumors with the exception of cases 2, 5 and 7 , which have already been described in the series by Asioli and coworkers (cases 2 and 5 in Asioli et al. (2014) described as cases 1 and 2, respectively; case 7 in Asioli et al. (2013) described as case 10).

All cases have been classified according to the previous diagnostic criteria ( $>30 \%$ hobnail features in the papillary carcinoma component) (Asioli et al. 2010). Four cases of classic papillary thyroid tumors and a normal pooled genomic DNA (DNA Female pool, Cod. G1521 Promega) were included as internal control for mutation analysis.

Inclusion criteria were the availability of representative hematoxylin and eosin sections for histological review and paraffin blocks for immunohistochemistry and molecular testing. Data regarding age, sex, presentation, gross pathology and post-surgery radiotherapy (iodine radiotherapy) were obtained by review of clinical files and/or contacting the referring clinician. The pathologic

Published by Bioscientifica Ltd 
staging was assigned according to the Seventh Edition Cancer Staging Manual of the American Joint Committee on Cancer (Edge et al. 2010).

Disease persistence, recurrence and metastases were evaluated by medical record review, imaging studies or histopathologic examination of resected or recurrent tumors and/or metastases.

\section{Immunohistochemistry}

Sections were cut at 4 microns and stained with antibodies against thyroid transcription factor 1 (TTF-1) (8G7G3/1,1:700; Dako), thyroglobulin (2H11/6E1cocktail, 1/50BRD; Zymed, Waltham, MA, USA), p53 (DO7,1:2000; Dako), mitochondria (JJ3-J, 1:100; Bio Genex, Fremont, CA, USA), Mucin 1 (MUC 1) (Ma695, 1:100; Novocastra, Milan, Italy), cytokeratin 7 (OB-TL12/30,1:200; Dako), E-cadherin (4A2C7, 1:100; Zymed), $\beta$-catenin (E-5, 1:200; Santa Cruz) and Ki-67 (MIB-1, 1:300; Dako). Immunoreactions were performed according to the standard automated immunohistochemical procedure (Ventana XT autostainer).

\section{Mutation analysis}

Mutation analysis workflow is summarized in Fig. 1. DNA from formalin-fixed paraffin-embedded tissues was purified using the Simple Prep kit (4bases SA, Manno, Switzerland). Mutation analysis was performed by deep sequencing for the following genes previously described to be involved in thyroid carcinogenesis (Cancer Genome Atlas Research Network 2014, Charles et al. 2014, Lee et al. 2015): KRAS (Exons 2-4), NRAS (Exons 2-4), HRAS (Exons 2, 3), BRAF (Exon 15), PIK3CA (Exons 10, 21), TP53 (Exons 4-9), NOTCH1 (Exons 26, 27), PTEN (Exons 5-8), CDKN2A (Exons 1, 2), EGFR (Exons 18-21), AKT1 (Exon 2), CTNNB1 (Exon 3) and hTERT (Promoter). Locus-specific amplicon libraries with tagged primers were generated using target enrichment kits (4bases SA). Oligo primers included overhang adapters based on Nextera sequence at the $5^{\prime}$, to be recognized by a second round of short PCR step to add Illumina (San Diego, CA, USA) P5/P7 sequencing adapters and sample-specific indices to samples. Six multiplex PCR tubes allowed parallel amplifications of all the above cited exons and hTERT promoter using Phusion II (Life Technologies), as a proofreading enzyme. Amplification products for each sample were mixed together and purified by AMPure XT (Agencourt-Beckman Coulter), quantified with the FluorometerQuantus (Promega) and then employed as template (100ng) for a second round

\section{Collection of cases and selection of tumor area}

$$
\downarrow
$$

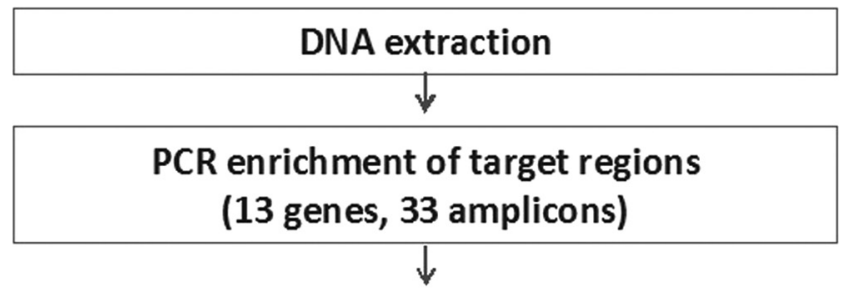

Library preparation: sample barcoding and pooling
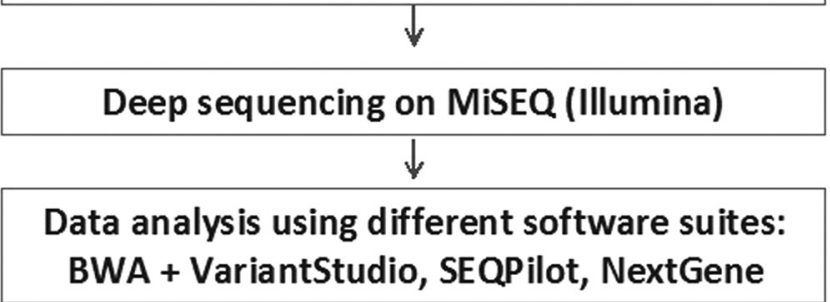

Figure 1

Mutational analysis work flow. Deep sequencing analysis involves the following steps.

of PCR (6 cycles) for barcoding (4bases SA). Amplicon products were purified with AgencourtAMPure XP beads, quantified with the FluorometerQuantus, pooled and loaded on MiSEQ (Illumina). Processed and quality-filtered reads from FASTQ files were analyzed with VariantStudio (Illumina), SEQPilot (JSI) and NextGene (SoftGenetics, State College, PA, USA). Only reads exceeding the Phred quality score of Q30 were retained for further bioinformatic analysis. Controls: DNA samples from normal pooled genomic DNA (Female pool, Cod. G1521 Promega), and cell lines known to carry a specific mutation were used as positive controls. Allele-specific locked nucleic acid quantitative PCR (ASLNAqPCR), as described previously (Morandi et al. 2012), was used as an orthogonal method to confirm the sensitivity and specificity of NGS results for BRAF (p.V600E) and KRAS (p.G12A, p.G12C, p.G12D, p.G12R, p.G12S, p.G12V and p.G13D) genes. Although the analytical sensitivity was detected to be less than $1 \%$, as confirmed by ASLNAqPCR, we decided to set the clinical sensitivity to $3 \%$ as previously described (Nikiforov et al. 2014); thus, only mutations detected to cross this threshold were reported.

\section{RET/PTC rearrangement}

The BRAF mutation-negative patients (5 patients) were further tested for RET/PTC rearrangement. RNA was

Published by Bioscientifica Ltd. 
extracted from the same paraffin blocks using RecoverAll kit (Life Technologies) as reported earlier (Asioli et al. 2010). In brief, RT-PCR for RET/PTC1 and RET/PTC3 was carried out using thyroid carcinoma with known RET/PTC1 and RET/PTC3 rearrangements as positive controls and omitting reverse transcriptase as negative controls. Primers used were as follows: RET/PTC1, 5'-ATT GTC ATC TCG CCG TTC-3' and RET/PTC3, 5'-TGG AGA AGA GAG GCT GTA TC-3'. Reverse primers were 5'-TGC TTC AGG ACG TTG AAC-3' for RET/PTC1 and 5'-CGT TGC CTT GAC TTT TC-3' for RET/PTC3. The housekeeping gene $P G K$ was used as an internal control.

\section{Statistical analysis}

Kaplan-Meier estimates of overall survival and disease-free survival were obtained. Univariate Cox regression analyses were used to compare the overall and disease-free survival between patients with and without a single mutation and between patients with and without multiple mutations. For multiple mutation analyses, we combined individuals with at least one BRAF, TP53 or PIK3CA mutation and compared them with the rest of the sample. A bootstrap procedure was used to obtain robust estimates of the standard errors for Cox regression analyses. Statistical analysis was performed using the Prism 4 (GraphPad) and IBM SPSS, version 20.0. The significance level was set at $P<0.05$.

\section{Results}

\section{Clinicopathological data and immunohistochemical profile}

The study cohort included 18 patients with HPTC. Clinical and histopathological features of the whole population are summarized in Table 1.

All patients underwent total thyroidectomy and cervical lymph nodes dissection, followed by radioiodine therapy (131I between 100 and $200 \mathrm{mCi}$ ).

Six out of 18 patients had a good response to radioactive iodine with good uptake and shrinkage of metastatic diseases, when present. A poor response (evidence of recurrence) after an average of 50 months (see below) was evidenced in five patients who had RAI-responded to initial treatment with thyroglobulin levels $<1 \mathrm{ng} / \mathrm{mL}$. The remaining 7 patients evidenced a poor response to RAI without uptake. The patients' thyroglobulin levels were available in all but one patient and ranged between $0.3 \mathrm{ng} / \mathrm{mL}$
Table 1 Clinical and histopathological characteristics of 18 hobnail papillary thyroid cancers.

\begin{tabular}{|c|c|c|}
\hline & Number of cases & Total (\%) \\
\hline \multicolumn{3}{|l|}{ Gender } \\
\hline Female & 14 & 77.8 \\
\hline Male & 4 & 22.2 \\
\hline \multicolumn{3}{|c|}{ Age (years) } \\
\hline$<45$ & 3 & 16.7 \\
\hline$\geq 45$ & 15 & 83.3 \\
\hline \multicolumn{3}{|c|}{ Tumor size $(\mathrm{cm})$} \\
\hline$\leq 4$ & 12 & 66.7 \\
\hline$>4$ & 6 & 33.3 \\
\hline \multicolumn{3}{|c|}{ Multifocality } \\
\hline Yes & 6 & 33.3 \\
\hline No & 12 & 66.7 \\
\hline \multicolumn{3}{|c|}{ Extra-thyroidal extension } \\
\hline Yes & 16 & 88.9 \\
\hline No & 2 & 11.1 \\
\hline \multicolumn{3}{|c|}{ Angiolymphatic invasion } \\
\hline Yes & 15 & 83.3 \\
\hline No & 3 & 16.7 \\
\hline \multicolumn{3}{|c|}{$\begin{array}{l}\text { Mitosis per } 10 \text { high-powered fields } \\
(400 \times)\end{array}$} \\
\hline$<3$ & 2 & 11.1 \\
\hline$\geq 3$ & 16 & 88.9 \\
\hline \multicolumn{3}{|l|}{$\mathrm{pT}$} \\
\hline 1 & 1 & 5.6 \\
\hline 2 & 1 & 5.6 \\
\hline 3 & 14 & 77.7 \\
\hline 4 & 2 & 11.1 \\
\hline \multicolumn{3}{|l|}{$\mathrm{pN}$} \\
\hline NO & 5 & 27.8 \\
\hline N1a & 2 & 11.1 \\
\hline $\mathrm{N} 1 \mathrm{~b}$ & 11 & 61.1 \\
\hline
\end{tabular}

and $102 \mathrm{ng} / \mathrm{mL}$ with a mean of $7.3 \mathrm{ng} / \mathrm{mL}$; median thyroglobulin levels showed a trend toward changing according to the radiological response (reduction, stabilization or progression).

Follow-up data were available for all patients with a mean of 40 months (range: 6-12 months). Five patients had recurrent disease after a mean of 50 months (range: 6-128 months) from the thyroidectomy. Eight patients developed metastatic disease, most commonly to the bone (5 cases), lung ( 2 cases) or mediastinum (1 case) after a mean of 68 months (range: 14-128 months) from the thyroidectomy. Recurrence and metastases to lymph nodes or distant organs showed a predominant hobnail pattern of growth similar to the primary tumor (Figs 2 and 3).

Four patients died of disease after a mean of 22 months. Seven patients remained alive with extensive disease after a mean follow-up of 49 months. Seven patients remained alive without disease after 42 months of follow-up.

The neoplastic cells showed variable thyroglobulin, TTF-1 and cytokeratin 7 immunoreactivity (Fig. 3). Ten out

Published by Bioscientifica Ltd 


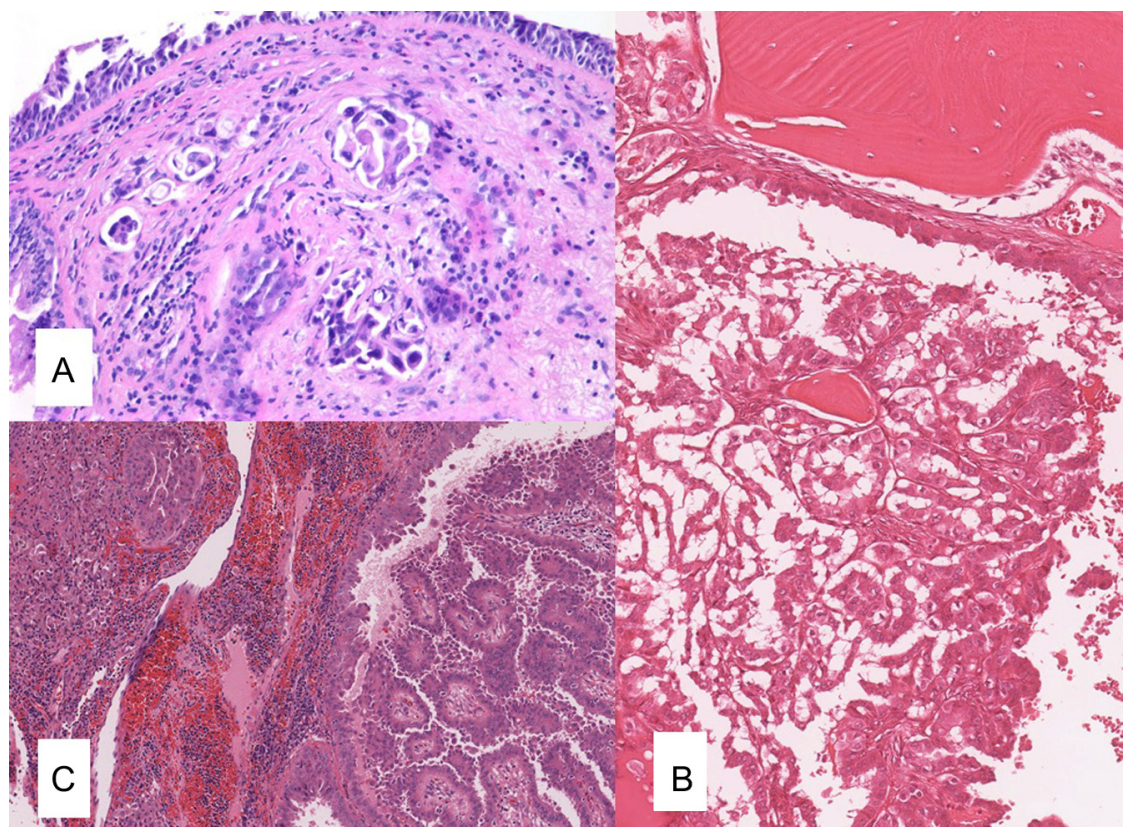

\section{Figure 2}

Metastasis to distant organs including lung (A) and bone (B) and to lymph node (C), maintained predominant hobnail pattern of growth similar to the primary tumor. A full colour version of this figure is available at http://dx.doi. org/10.1530/ERC-16-0546. of 18 cases showed more than $25 \%$ of the neoplastic cell nuclei positive for p53 (Fig. 3) in the primary thyroid tumor and in lymph nodes metastases. E-cadherin and $\beta$-catenin are present in the basolateral or lateral site of neoplastic cells, and in three cases, $\beta$-catenin also showed nuclear co-expression in few cells $(<5 \%)$. MUC1 staining showed the characteristic 'inside-out pattern' highlighted by hobnail features of the tumor cells. Only one case (case 12) showed focal positivity (20\%) for the antimitochondria antibody. The mean of proliferative index Ki67 was $12 \%$.

\section{Molecular profile}

DNA from all samples was successfully amplified in multiplex PCR, and an adequate library for NGS was obtained in all 18 patients. The mean read length was 183 base pairs and a mean coverage depth of 1203 was achieved, with 99.5\% target bases covered more than $100 \times$. No mutations were detected for normal pooled genomic DNA (Promega).

Pathogenic mutations in the 13 genes were found in 15 of 18 (83.3\%) patients (Table 2). Of the

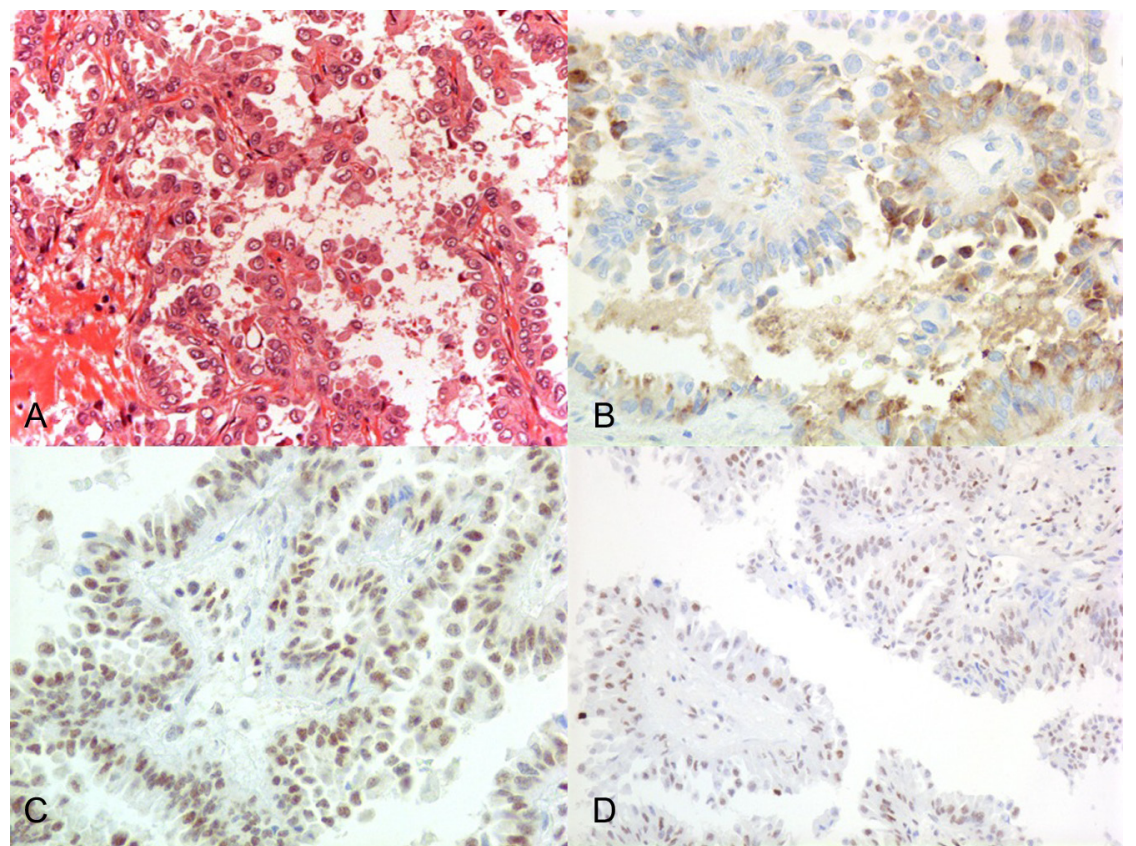

Figure 3

A HPTC case harboring BRAF, TP53 and PIK3A mutations shows papillary and micropapillary patterns of growth (A) with focal positivity for thyroglobulin (B), nuclear staining for TTF1 (C) and nuclear overexpression of p53 protein (D). A full colour version of this figure is available at http://dx.doi.org/10.1530/ERC-16-0546. 


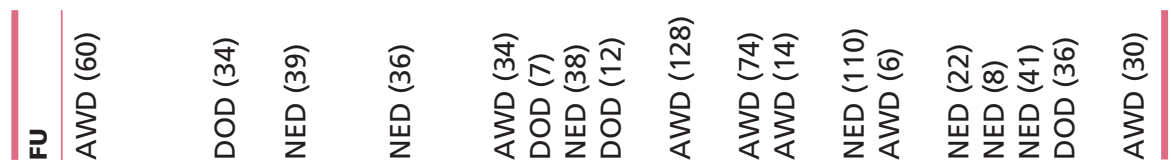
|

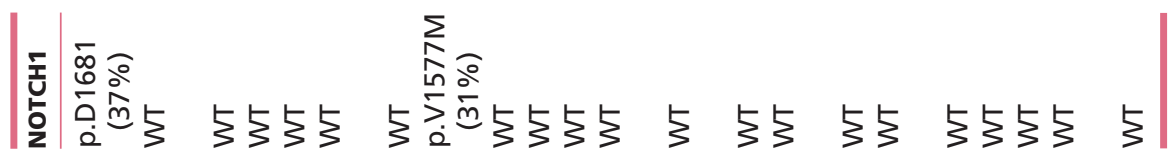

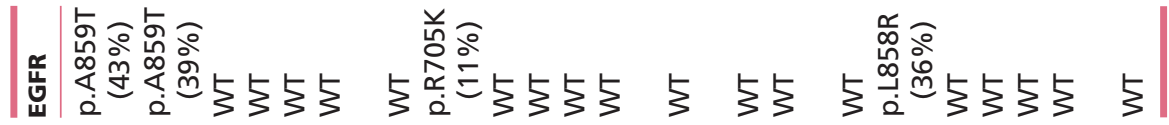

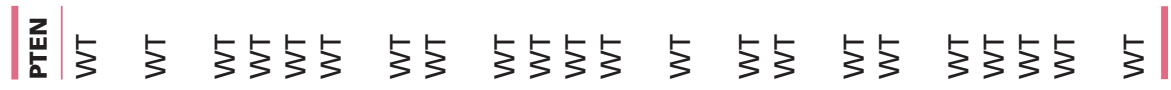

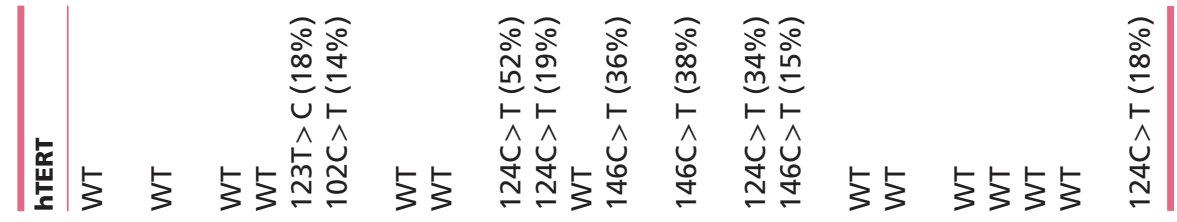

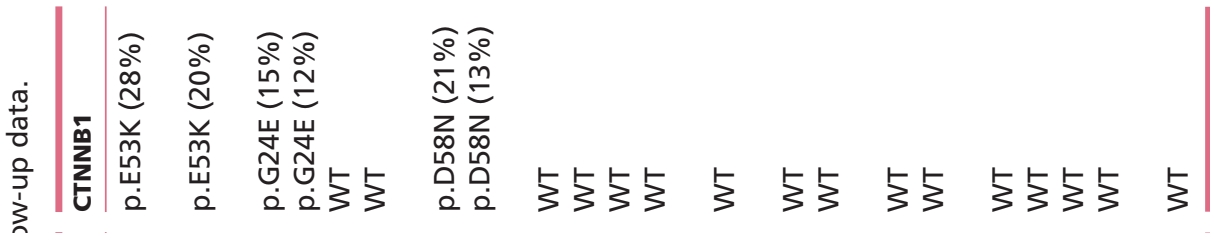

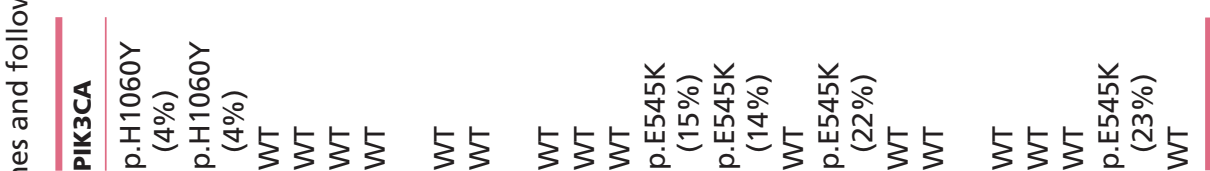

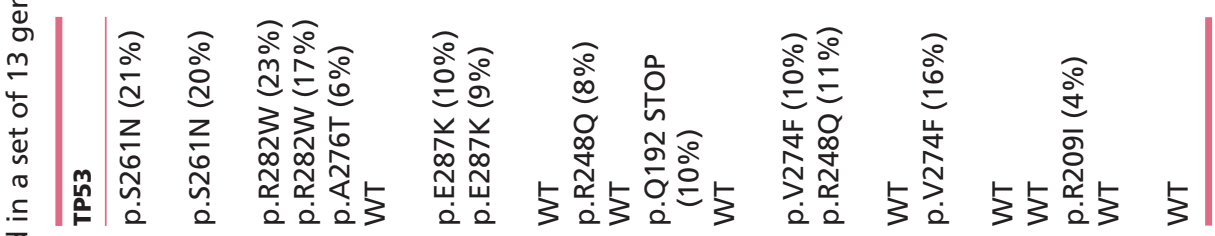

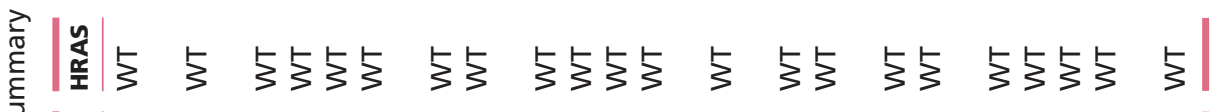

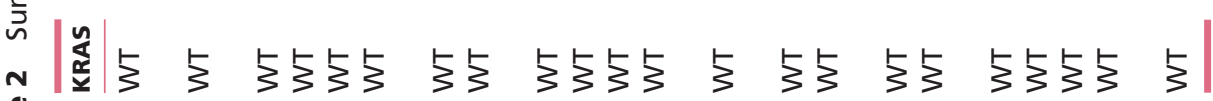

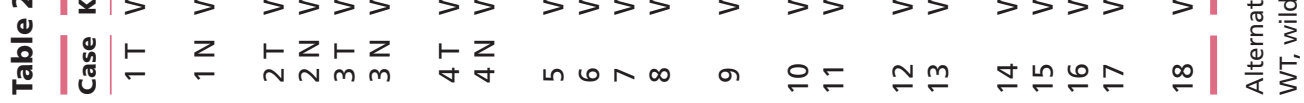


fifty-eight mutations found in these 22 cases of HPTC, including eighteen primary tumors and four lymph node metastases, forty-eight were missense, one was a premature stop codon and the remaining ones are singlebase substitutions at the promoter of hTERT. Forty-six out of 49 genetic alterations found in coding regions are recorded in the COSMIC databases. The remaining three were not previously described in solid tumors and they were detected in $A K T 1$ (p.D32N, p.G16E) and NOTCH1 (p.D1681N) genes. BRAF and TP53 mutations were by far the most common genetic alterations in HPTC patients $(72.7 \%$ and $55.6 \%$, respectively). BRAF p.V600E mutation was the most common alteration found in our series. All the 15 samples positive for p.V600E by NGS were confirmed by ASLNAqPCR. Additionally, we identified a rarer variant, the p.A598V (case 3), which has been recorded in COSMIC database, and it was found pathogenic in thyroid cancer. Only five of 18 primary HPTC cases included in this study did not harbor any pathogenic mutations in BRAF gene.

TP53 mutations were detected in ten out of 18 primary HPTC cases, all of which have been included in the COSMIC and TP53 database (IARC) and were considered pathogenic. Seven primary HPTC cases evidenced the simultaneous presence of both BRAF and TP53 mutations.

PIK3CA mutations were found in five primary HPTC cases. hTERT was investigated from position -191 to -64 upstream from the ATG start site. In our series, eight cases out of 18 primary HPTC cases (44.4\%) showed mutations mostly in the two damaging hot spots, at positions -124 and -146 . CTNNB1 was found to be mutated in three primary HPTC cases (16.7\%), EGFR in two (11.1\%) and AKT1 and NOTCH1 in one case (5.5\%). Mutations of CKDN2A and PTEN were not detected. The mutational pattern in primary tumors and metastasis was for the most part maintained for cases 1 and 2, except for NOTCH1 and $A K T 1$ in case 1 , indicating clonality. The mutation frequency of $B R A F$ was very close to $50 \%$ for cases 1 and 2 both for primary and metastasis. Cases 4-6, 8-12 and 17-18 showed a mutation frequency within a range between $7 \%$ and $30 \%$. Among various gene mutations detected, those of BRAF presented the highest frequency supporting the fundamental role of this gene in HPTC variant and the presence of these mutations in the bulk of the tumor.

In case 3 , only metastasis was involved in the rare $B R A F$ p.A598V and $A K T 1$ p.G16E with $15 \%$ and $18 \%$ mutation allele frequency, respectively. Additionally TP53 p.A276P (7\% frequency) was detected only in

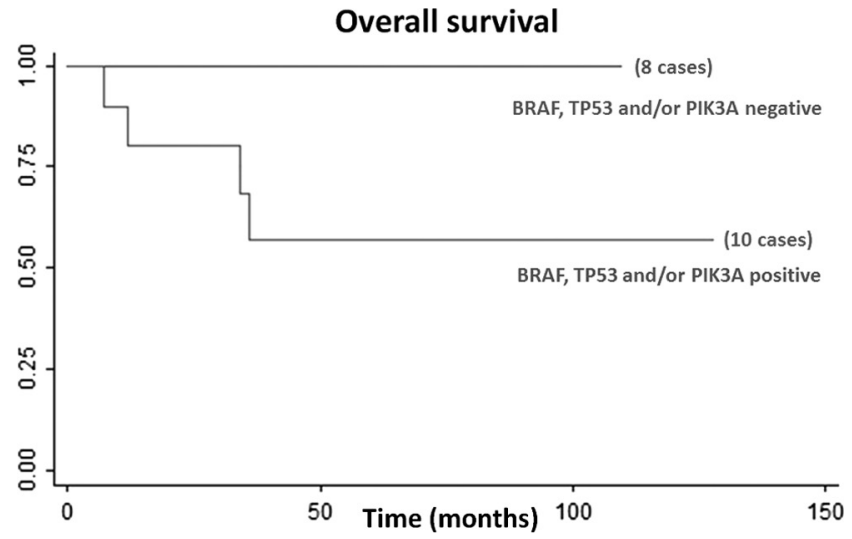

Figure 4

Kaplan-Meier estimates of overall survival in 10 HPTC with BRAF, TP53 and/or PIK3A mutation vs the rest of sample $(N=8)$.

primary lesion, and two different mutations were identified for TERT, proving tumor heterogeneity in this case (Table 2).

One patient showed mutations only for BRAF gene (p.V600E) in primary HPTC (case 12).

In the five patients without BRAF mutation, the RET/PTC rearrangement analyses were negative for RET/PTC1 and RET/PTC3 rearrangements in three patients. The remaining two patients did not have amplifiable cDNA.

Figure 4 shows the Kaplan-Meier estimates of overall survival in 10 HPTC with BRAF, TP53 and/or PIK3A mutation vs the rest of sample $(N=8)$.

Table 3 summarizes the results of univariate Cox regression analyses with bootstrap procedure that indicate a significantly increased mortality risk in patients harboring BRAF mutation and BRAF mutation associated with TP53 and/or PIK3CA mutations. Specifically, in patients harboring $B R A F$ mutation, the mortality risk was 5.4-fold compared with those not harboring the mutation, and it was 6.6-fold in those harboring the combination of mutations. None of the gene alterations considered, including BRAF, TP53, PIK3CA, hTERT and CTNNB1, was associated with a shorter disease-free survival.

Table 3 Hazard ratios (HR) and 95\% confidence intervals (Cl) estimated using a bootstrap procedure.

\begin{tabular}{|c|c|c|c|c|}
\hline \multirow{2}{*}{$\frac{\text { Genes }}{\text { BRAF }}$} & \multirow{2}{*}{$\frac{\text { HR }}{5.394}$} & \multicolumn{2}{|c|}{$95 \% \mathrm{Cl}$} & \multirow{2}{*}{$\frac{\boldsymbol{P}}{0.001}$} \\
\hline & & 4.204 & 6.917 & \\
\hline TP53 & 1.596 & 0.190 & 13.404 & 0.233 \\
\hline PIK3CA & 1.500 & 0.154 & 14.622 & 0.218 \\
\hline CTNNB1 & 1.095 & 0.112 & 10.745 & 0.907 \\
\hline hTERT & 1.207 & 0.147 & 9.924 & 0.661 \\
\hline BRAF and (TP53 and/or PK3CA) & 6.605 & 4.217 & 10.348 & 0.001 \\
\hline
\end{tabular}

Published by Bioscientifica Ltd.
(C) 2017 Society for Endocrinology Printed in Great Britain 


\section{Discussion}

As the original description of HPTC as a separate entity among malignant thyroid tumors, several tumor series have been reported (Bellevicine et al. 2012, Asioli et al. 2013, 2014, Amacher et al. 2015, Lee et al. 2015). In this study, we performed immunohistochemical and molecular analyses of a large series of HPTCs selected using the strict previously described criteria (Asioli et al. 2013). The immunohistochemical profile of our series is consistent with data previously reported (Asioli et al. 2010). The peculiar and distinctive immunophenotype shown by HPTC, which consists of TTF-1 and thyroglobulin expressions (markers expressed in well-differentiated thyroid cancer and lost in ATC) and in the simultaneous overexpression of p53 protein (marker overexpressed in ATC), was confirmed from the molecular analysis for the gene TP53.

TP53 pathogenetic mutations were detected in 10 out of 18 primary HPTC cases, all of which have been included in the COSMIC and TP53 database (IARC) and were considered pathogenic. All ten cases showed more than $25 \%$ of the neoplastic cell nuclei positive for p53 in the primary thyroid tumor, and we did not observe any peculiar p53 immunohistochemistry correlation with mutation status in either distribution and/or intensity. Nikiforova and coworkers (Nikiforova et al. 2013), using the sensitive NGS technique, reported $\beta$-catenin follicular thyroid carcinoma oncocytic variant, and in $29.6 \%$ of ATC. They reported an increasing frequency of TP53 mutations in dedifferentiating thyroid carcinomas. The same study reported the presence of mutations for PIK3CA in $11.1 \%$ of PTC and ATC and one case of ATC and one case of PTC showed concurrent mutation in PIK3CA and TP53 (Nikiforova et al. 2013).

In addition, neoplastic cells of HPTC showed expression of E-cadherin and $\beta$-catenin in the basolateral or lateral site, and in three cases, we found mutations of CTNNB1 and $\beta$-catenin nuclear co-expression in few neoplastic cells $(<5 \%)$. Molecular data in our series found CTNNB1 gene mutated in three patients (16.7\%), both in primary and in metastatic tumor, confirming our previous data (Asioli et al. 2010). Considering CTNNB1 gene mutations, Nikiforova and coworkers (Nikiforova et al. 2013) found only one positive case of ATC. Basolateral or lateral or nuclear expression of $\beta$-catenin is considered one of molecular event involved in epithelial-mesenchymal transition (EMT). EMT is an established pathologic mechanism in epithelial tumor progression, local invasion and metastasis that has been implicated in HPTC (Masago et al. 2009). The elucidation of molecular mechanisms that govern EMT will offer new approaches and targets to restrain metastasis.

Our molecular results identify multiple alterations including an initial driver (BRAF) plus second hits (TP53, TERT and PIK3CA) that is not novel or unique to HPTC (Cancer Genome Atlas Research Network 2014), but this molecular subtype could be useful to better identify HPTCs that reflect its underlying signaling and differentiation properties, which has the potential to improve its pathological classification. Indeed, BRAF mutations were detected mostly in the bulk of tumor with a higher variant allele frequency (VAF) than those found in other genes such as PIK3CA, TP53 or CTNNB1 (Table 2). This mutational pattern revealed the fundamental role of $B R A F$ as a driver, followed by TP53, and in a more restricted number of cases by PIK3CA, CTNNB1 and hTERT.

For instance, the primary tumor of case 1 showed a VAF of $42 \%$ for BRAF p.V600E, $43 \%$ for EGFR p.A859T and $37 \%$ for NOTCH1 p.D1681. This means that almost all the bulk of this tumor shared these mutations that can be considered early events in its evolution. Other mutations detected in TP53, CTNNB1, AKT and PIK3CA displayed lower VAF (Table 2), a typical sign of later events that involved a more limited number of tumor cells. In 3 out of 4 cases where metastasis was available (cases 1, 2 and 4), the same mutation pattern with a similar VAF was present in both primary tumor and metastasis, revealing that presumably these mutations were maintained over the time course of the tumor evolution, except for NOTCH1 and AKT1mutations. Although our series is made of a limited number of cases, it may reveal that in these patients a single clone gave rise to lymph node metastasis maintaining driver mutations in BRAF, TP53 or PIK3CA, and mutations in these genes are related to a more aggressive behavior. Activating mutations of BRAF and PIK3CA in cooperation has been described recently in anaplastic carcinoma (Charles et al. 2014), and treating these tumors with selective or multi-tyrosine kinase inhibitor such as sorafenib, may improve clinical outcome (Brose et al. 2014). Only case 3 exhibited a completely different mutational pattern between primary tumor and metastasis with 4 unmatched mutations. This means that the primary tumor is unrelated to lymph node metastasis, which may be derived from an occult undetected primary tumor or by a micro-clone not present in cells collected from the selected tumor tissue. The combined mutations in BRAF, TP53 and/or PIK3CA genes by molecular profiling seem to be clinically relevant for the prognostic stratification

Published by Bioscientifica Ltd. 
and treatment of these patients. BRAF p.V600E and the rarer variant p.A598V were found in most cases of our series. Accordingly, BRAF p.V600E mutation is the most prevalent point mutation in PTC being present in 36-69\% of cases (Trovisco et al. 2005), and it was detected almost exclusively in papillary or mixed follicular/papillary thyroid carcinomas (Trovisco et al. 2005). Currently, poor prognostic indicators, such as extra-thyroid extension (Nikiforova et al. 2003), metastasis, higher tumor staging, tumor size and recurrence (Xing et al. 2015), were found associated with BRAF mutations as well as its association with a decrease in expression of several 'thyroid-specific genes' or 'iodine-handling genes'. Previous studies reported that low radioactive iodine avidity are associated to BRAF mutations in vitro and in vivo (Chakravarty et al. 2011), and this should be the case for HPTC.

In the present series, RET/PTC1 and RET/PTC3 rearrangements were not detected in BRAF V600E-negative tumors, confirming our previous data (Asioli et al. 2010). Recently, Lubitz and coworkers (Lubitz et al. 2014), using SNaPshot analysis reported that two BRAF p.V600Enegative tumors out of twelve HPTC cases carried the RET/PTC1 gene rearrangement as well as Ieni and coworkers (Ieni et al. 2016), using RT-PCR analysis, among the 4 BRAF p.V600E-negative cases out of eight HPTC cases reported in their study, only one exhibited RET/PTC1 gene rearrangement.

hTERT mutations were found in 8 out of 18 patients with HPTC (44.4\%) in this study, and similar data for poorly differentiated carcinomas (29\%) and anaplastic carcinomas (33.3\%) were described by other groups with a significant association with disease-specific mortality (Melo et al. 2014). The mutations in the current study occurred mostly in two hotspot positions, mapped at $-124 \mathrm{bp}$ and $-146 \mathrm{bp}$ upstream from the ATG start site. These two hotspots were reported to confer enhanced $h T E R T$ promoter activity, generating a consensus binding site (GGAA) for ETS transcription factors within the $h T E R T$ promoter region in melanoma (Horn et al. 2013).

Interestingly, we found four cases with mutations in the EGFR gene, three of which also had BRAF mutation: one case harbored the most common pathogenic variant p.L858R, which was found to be annotated in the COSMIC database related not only for lung carcinomas but even to six thyroid tissues of origin. The other two EGFR variants (p.A859T and p.R705K) were annotated in COSMIC database for lung carcinoma and urinary tract and were both considered probably damaging by PolyPhen. Previous studies have failed to identify the EGFR-activating mutations in thyroid carcinomas (only 3.2\% mutated cases) by direct sequencing (Mitsiades et al. 2006, Lee et al. 2007); however, Masago and coworkers (Masago et al. 2009) reported 7 positive cases out of 23 primary PTCs for exons 19 and 21 using more sensitive techniques (PNA-LNA clump).

Among these seven positive cases of Masago's series (Masago et al. 2009), three were reported having concurrent BRAF mutation and one patient with PTC whose tumor harbored the EGFR-activating mutation, showed a marked response to an EGFR-tyrosine-kinase inhibitor (Gefitinib).

In this study, we did not find any pathogenic variants of KRAS, NRAS and HRAS. As previously described, RAS mutations are more prevalent and seem to be more relevant as a prognostic indicator in follicular lesions than those in classic PTC (Sobrinho-Simoes et al. 2008, Giordano et al. 2014).

To date, tyrosine kinase inhibitors (TKIs) represent a new approach to the treatment of advanced cases of RAI-R DTC. Recently, sorafenib (ClinicalTrial.gov identifier NTC00984282) and lenvatinib (ClinicalTrial. gov identifier NCT01321554) have been approved for DTC. Vemurafenib (ClinicalTrial.gov identifier NCT01286753) is studied for the treatment of RAI-R, progressive, metastatic or unresectable PTC patients with BRAF p.V600 mutation with a good progressionfree survival in both cohort of patients (TKI treatment naïve or TKI treated) (Brose et al. 2014), data recently confirmed by Dadu and coworkers (Dadu et al. 2015). Pazopanib, which targets VEGFR, PDGFR, c-KIT and other kinases, is studied in a cohort of RAI-R and rapidly progressing metastatic DTC (ClinicalTrial.gov identifier NCT00625846), with a good partial response (Bible et al. 2010). The future steps in treatment of advanced thyroid cancers should be targeting different molecular pathways and combined therapies to obtain a synergistic effect to control the disease and to reduce drug toxicities and side effects.

In conclusion, despite a significant prevalence of BRAF mutation, more than $70 \%$ of HPTCs in our series showed concurrent mutations of other genes such as TP53, PIK3CA, CTNNB1 and hTERT, in contrast to classic PTC. The molecular portrait of HPTC reported in the present study further outlines the complex pathogenetic mechanisms of this variant and may offer the tumor genotype of HPTC to direct new strategies for a personalized treatment of these tumors. 


\section{Declaration of interest}

The authors declare that there is no conflict of interest that could be perceived as prejudicing the impartiality of the research reported.

\section{Funding}

This work was supported by the Ricerca Fondamentale Orientata (RFO) 2014, University of Bologna, Italy to S A.

\section{Author contribution statement}

Luca Morandi designed the experiments, performed mutational analysis and wrote the manuscript; Leonardo Caporali and Alberto Righi performed immunohistochemical and mutational analyses; Francesca Maletta, Fabio Pagni, Sabrina Rossi and Anna Sapino collected HPTC cases and did the revision of histological preparations; Marco Gallo recruited clinical and follow-up data of the HPTC patients; Paola Rucci did statistical analysis; Ricardo V Lloyd and Sofia Asioli coordinated the research group, they performed the revision of histological preparations, the supervision of molecular analysis and they wrote the manuscript.

\section{Acknowledgements}

This work was presented in part at the 105th United States and Canadian Academy of Pathology Annual Meeting, Seattle, Washington, USA, 12-18 March 2016.

\section{References}

Abubaker J, Jehan Z, Bavi P, Sultana M, Al-Harbi S, Ibrahim M, Al-Nuaim A, Ahmed M, Amin T, Al-Fehaily M, et al. 2008 Clinicopathological analysis of papillary thyroid cancer with PIK3CA alterations in a Middle Eastern population. Journal of Clinical Endocrinology and Metabolism 93 611-618. (doi:10.1210/jc.2007-1717)

Amacher AM, Goyal B, Lewis JS Jr, El-Mofty SK \& Chernock RD 2015 Prevalence of a hobnail pattern in papillary, poorly differentiated, and anaplastic thyroid carcinoma: a possible manifestation of highgrade transformation. American Journal of Surgical Pathology 39 260-265. (doi:10.1097/PAS.0000000000000329)

Asioli S, Erickson LA, Sebo TJ, Zhang J, Jin L, Thompson GB \& Lloyd RV 2010 Papillary thyroid carcinoma with prominent hobnail features: a new aggressive variant of moderately differentiated papillary carcinoma. A clinicopathologic, immunohistochemical, and molecular study of eight cases. American Journal of Surgical Pathology 34 44-52. (doi:10.1097/PAS.0b013e3181c46677)

Asioli S, Erickson LA, Righi A \& Lloyd RV 2013 Papillary thyroid carcinoma with hobnail features: histopathologic criteria to predict aggressive behavior. Human Pathology 44 320-328. (doi:10.1016/j. humpath.2012.06.003)

Asioli S, Maletta F, Pagni F, Pacchioni D, Vanzati A, Mariani S, Palestini N, Lloyd RV \& Sapino A 2014 Cytomorphologic and molecular features of hobnail variant of papillary thyroid carcinoma: case series and literature review. Diagnostic Cytopathology 42 78-84. (doi:10.1002/dc.23028)

Bellevicine C, Cozzolino I, Malapelle U, Zeppa P \& Troncone G 2012 Cytological and molecular features of papillary thyroid carcinoma with prominent hobnail features: a case report. Acta Cytologica 56 560-564. (doi:10.1159/000338395)

Bible KC, Suman VJ, Molina JR, Smallridge RC, Maples WJ, Menefee ME, Rubin J, Sideras K, Morris JC 3rd, McIver B, et al. 2010 Efficacy of pazopanib in progressive, radioiodine-refractory, metastatic differentiated thyroid cancers: results of a phase 2 consortium study. Lancet Oncology 11 962-972. (doi:10.1016/S1470-2045(10)70203-5)

Brose MS, Nutting CM, Jarzab B, Elisei R, Siena S, Bastholt L, de la Fouchardiere C, Pacini F, Paschke R, Shong YK, et al. 2014 Sorafenib in radioactive iodine-refractory, locally advanced or metastatic differentiated thyroid cancer: a randomised, double-blind, phase 3 trial. Lancet 384 319-328. (doi:10.1016/S01406736(14)60421-9)

Cancer Genome Atlas Research Network 2014 Integrated genomic characterization of papillary thyroid carcinoma. Cell 159 676-690. (doi:10.1016/j.cell.2014.09.050)

Chakravarty D, Santos E, Ryder M, Knauf JA, Liao XH, West BL, Bollag G, Kolesnick R, Thin TH, Rosen N, et al. 2011 Small-molecule MAPK inhibitors restore radioiodine incorporation in mouse thyroid cancers with conditional BRAF activation. Journal of Clinical Investigation 121 4700-4711. (doi:10.1172/JCI46382)

Charles RP, Silva J, Iezza G, Phillips WA \& McMahon M 2014 Activating BRAF and PIK3CA mutations cooperate to promote anaplastic thyroid carcinogenesis. Molecular Cancer Research 12 979-986. (doi:10.1158/1541-7786.MCR-14-0158-T)

Chung YJ, Lee JS, Park SY, Park HJ, Cho BY, Park SJ, Lee SY, Kang KH \& Ryu HS 2013 Histomorphological factors in the risk prediction of lymph node metastasis in papillary thyroid carcinoma. Histopathology 62 578-588. (doi:10.1111/his.12025)

Dadu R, Shah K, Busaidy NL, Waguespack SG, Habra MA, Ying AK, Hu MI, Bassett R, Jimenez C, Sherman SI, et al. 2015 Efficacy and tolerability of vemurafenib in patients with $\operatorname{BRAF}(V 600 E)$-positive papillary thyroid cancer: M.D. Anderson Cancer Center off label experience. Journal of Clinical Endocrinology and Metabolism 100 E77-E81. (doi:10.1210/jc.2014-2246)

Edge S, Byrd DR, Compton CC, Fritz AG, Greene FL \& Trotti A 2010 Cancer Staging Handbook (From the AJCC Cancer Staging Manual), 7th edition. New York, NY, USA: Springer Science+Business Media LLC.

Giordano TJ, Beaudenon-Huibregtse S, Shinde R, Langfield L, Vinco M, Laosinchai-Wolf W \& Labourier E 2014 Molecular testing for oncogenic gene mutations in thyroid lesions: a case-control validation study in 413 postsurgical specimens. Human Pathology 45 1339-1347. (doi:10.1016/j.humpath.2014.03.010)

Haugen BR, Alexander EK, Bible KC, Doherty GM, Mandel SJ, Nikiforov YE, Pacini F, Randolph GW, Sawka AM, Schlumberger M, et al. 20162015 American Thyroid Association Management guidelines for adult patients with thyroid nodules and differentiated thyroid cancer: the American Thyroid Association guidelines task force on thyroid nodules and differentiated thyroid cancer. Thyroid 26 1-133. (doi:10.1089/thy.2015.0020)

Horn S, Figl A, Rachakonda PS, Fischer C, Sucker A, Gast A, Kadel S, Moll I, Nagore E, Hemminki K, et al. 2013 TERT promoter mutations in familial and sporadic melanoma. Science 339 959-961. (doi:10.1126/science.1230062)

Kakudo K, Bai Y, Liu Z, Li Y, Ito Y \& Ozaki T 2012 Classification of thyroid follicular cell tumors: with special reference to borderline lesions. Endocrine Journal 59 1-12. (doi:10.1507/endocrj.EJ11-0184)

Ieni A, Barresi V, Cardia R, Licata L, Di Bari F, Benvenga S \& Tuccari G 2016 The micropapillary/hobnail variant of papillary thyroid carcinoma: a review of series described in the literature compared to a series from one southern Italy pathology institution. Reviews in Endocrine and Metabolic Disorders 17 1-7. (doi:10.1007/s11154-0169398-4)

Lee DH, Lee GK, Kong SY, Kook MC, Yang SK, Park SY, Park SH, Keam B, Park DJ, Cho BY, et al. 2007 Epidermal growth factor receptor status in anaplastic thyroid carcinoma. Journal of Clinical Pathology 60 881-884. (doi:10.1136/jcp.2006.041251)

Lee YS, Kim Y, Jeon S, Bae JS, Jung SL \& Jung CK 2015 Cytologic, clinicopathologic, and molecular features of papillary thyroid carcinoma with prominent hobnail features: 10 case reports and
(C) 2017 Society for Endocrinology Printed in Great Britain 
systematic literature review. International Journal of Clinical and. Experimental Pathology 8 7988-7997.

Lino-Silva LS, Dominguez-Malagon HR, Caro-Sanchez CH \& Salcedo-Hernández RA 2012 Thyroid gland papillary carcinomas with 'micropapillary pattern,' a recently recognized poor prognostic finding: clinicopathologic and survival analysis of 7 cases. Human Pathology 43 1596-1600. (doi:10.1016/j.humpath.2011.10.027)

Liu Z, Hou P, Ji M, Guan H, Studeman K, Jensen K, Vasko V, El-Naggar AK \& Xing M 2008 Highly prevalent genetic alterations in receptor tyrosine kinases and phosphatidylinositol 3-kinase/akt and mitogen-activated protein kinase pathways in anaplastic and follicular thyroid cancers. Journal of Clinical Endocrinology and Metabolism 93 3106-3116. (doi:10.1210/jc.2008-0273)

LiVolsi VA, Albores-Saavedra J, Asa SL, DeLellis RA, Lloyd RV, Heitz PU, \& Eng C 2004 Papillary carcinoma. In World Health Organization Classification of Tumours. Pathology and Genetics. Tumours of Endocrine Organs, pp. 57-66. Lyon, France: IARC Press.

Lubitz CC, Economopoulos KP, Pawlak AC, Lynch K, Dias-Santagata D, Faquin WC \& Sadow PM 2014 Hobnail variant of papillary thyroid carcinoma: an institutional case series and molecular profile. Thyroid 24 958-965. (doi:10.1089/thy.2013.0573)

Masago K, Asato R, Fujita S, Hirano S, Tamura Y, Kanda T, Mio T, Katakami N, Mishima M \& Ito J 2009 Epidermal growth factor receptor gene mutations in papillary thyroid carcinoma. International Journal of Cancer 124 2744-2749. (doi:10.1002/ijc.24250)

Mathur A, Moses W, Rahbari R, Khanafshar E, Duh QY, Clark O \& Kebebew E 2011 Higher rate of BRAF mutation in papillary thyroid cancer over time: a single-institution study. Cancer 117 4390-4395. (doi:10.1002/cncr.26072)

Melo M, da Rocha AG, Vinagre J, Peixoto J, Tavares C, Celestino R, Almeida A, Salgado C, Eloy C, Castro P, et al. 2014 TERT promoter mutations are a major indicator of poor outcome in differentiated thyroid carcinomas. Journal of Clinical Endocrinology and Metabolism 99 E754-E765. (doi:10.1210/jc.2013-3734)

Mitsiades CS, Kotoula V, Poulaki V, McMullan C, Negri J, Fanourakis G, Tseleni-Balafouta S, Ain KB \& Mitsiades N 2006 Epidermal growth factor receptor as a therapeutic target in human thyroid carcinoma: mutational and functional analysis. Journal of Clinical Endocrinology and Metabolism 91 3662-3666. (doi:10.1210/jc.2006-0055)

Morandi L, de Biase D, Visani M, Cesari V, De Maglio G, Pizzolitto S, Pession A \& Tallini G 2012 Allele specific locked nucleic acid quantitative PCR (ASLNAqPCR): an accurate and cost-effective assay to diagnose and quantify KRAS and BRAF mutation. PLOS ONE 7 E36084. (doi:10.1371/journal.pone.0036084)

Motosugi U, Murata S, Nagata K, Yasuda M \& Shimizu M 2009 Thyroid papillary carcinoma with micropapillary and hobnail growth pattern: a histological variant with intermediate malignancy? Thyroid 19 535-537. (doi:10.1089/thy.2008.0271)

Nikiforov YE, Carty SE, Chiosea SI, Coyne C, Duvvuri U, Ferris RL, Gooding WE, Hodak SP, LeBeau SO, Ohori NP, et al. 2014 Highly accurate diagnosis of cancer in thyroid nodules with follicular neoplasm/suspicious for a follicular neoplasm cytology by ThyroSeq v2 next-generation sequencing assay. Cancer 120 3627-3634. (doi:10.1002/cncr.29038)

Nikiforova MN, Kimura ET, Gandhi M, Biddinger PW, Knauf JA, Basolo F, Zhu Z, Giannini R, Salvatore G, Fusco A, et al. 2003 BRAF mutations in thyroid tumors are restricted to papillary carcinomas and anaplastic or poorly differentiated carcinomas arising from papillary carcinomas. Journal of Clinical Endocrinology and Metabolism 88 5399-5404. (doi:10.1210/jc.2003-030838)

Nikiforova MN, Wald AI, Roy S, Durso MB \& Nikiforov YE 2013 Targeted next-generation sequencing panel (ThyroSeq) for detection of mutations in thyroid cancer. Journal of Clinical Endocrinology and Metabolism 98 E1852-E1860. (doi:10.1210/ jc.2013-2292)

Romei C \& Elisei R 2012 RET/PTC translocations and clinicopathological features in human papillary thyroid carcinoma. Frontiers in Endocrinology 3 54. (doi:10.3389/fendo.2012.00054)

Santarpia L, El-Naggar AK, Cote GJ, Myers JN \& Sherman SI 2008 Phosphatidylinositol 3-kinase/akt and ras/raf-mitogen-activated protein kinase pathway mutations in anaplastic thyroid cancer. Journal of Clinical Endocrinology and Metabolism 93 278-284. (doi:10.1210/jc.2007-1076)

Schwock J, Desai G, Devon KM, Mete O \& Dubé V 2015 Hobnail-variant of papillary thyroid carcinoma in liquid-based cytology. Diagnostic Cytopathology 43 990-992. (doi:10.1002/dc.23338)

Smallridge RC, Marlow LA \& Copland JA 2009 Anaplastic thyroid cancer: molecular pathogenesis and emerging therapies. EndocrineRelated Cancer 16 17-44. (doi:10.1677/ERC-08-0154)

Sobrinho-Simoes M, Maximo V, Rocha AS, Trovisco V, Castro P, Preto A, Lima J \& Soares P 2008 Intragenic mutations in thyroid cancer. Endocrinology Metabolism Clinics of North America 37 333-362. (doi:10.1016/j.ecl.2008.02.004)

Trovisco V, Soares P, Preto A, de Castro IV, Lima J, Castro P, Máximo V, Botelho T, Moreira S, Meireles AM, et al. 2005 Type and prevalence of BRAF mutations are closely associated with papillary thyroid carcinoma histotype and patients' age but not with tumour aggressiveness. Virchows Archiv 446 589-595. (doi:10.1007/s00428005-1236-0)

Viola D, Valerio L, Molinaro E, Agate L, Bottici V, Biagini A, Lorusso L, Cappagli V, Pieruzzi L \&Giani C 2016 Treatment of advanced thyroid cancer with targeted therapies: ten years of experience. EndocrineRelated Cancer 23 185-205. (doi:10.1530/ERC-15-0555)

Xing M, Alzahrani AS, Carson KA, Shong YK, Kim TY, Viola D, Elisei R, Bendlová B, Yip L, Mian C, et al. 2015 Association between BRAF V600E mutation and recurrence of papillary thyroid cancer. Journal of Clinical Oncology 33 42-50. (doi:10.1200/ JCO.2014.56.8253)

Yang GC, Fried K \& Scognamiglio T 2013 Cytological features of clear cell thyroid tumors, including a papillary thyroid carcinoma with prominent hobnail features. Diagnostic Cytopathology 41 757-761. (doi:10.1002/dc.22935)

Received in final form 18 December 2016

Accepted 6 January 2017

Accepted Preprint published online 6 January 2017
() 2017 Society for Endocrinology Printed in Great Britain
Published by Bioscientifica Ltd. 